\title{
Enzymtechnologie
}

\section{Organisch-chemisch stabilisierte Enzyme für eine nachhaltige Wirtschaft}

SASKIA NEUBACHER, SVEN HENNIG, TOM N. GROSSMANN

DEPARTMENT OF CHEMISTRY AND PHARMACEUTICAL SCIENCES, VRIJE UNIVERSITEIT AMSTERDAM, NIEDERLANDE

Advances in protein engineering have made the industrial use of enzymes feasible, though, a more wide-spread application is hampered by the limited stability of enzymes under often required harsh conditions. We developed a protein stabilization technology that involves the in situ cyclization of proteins (INCYPRO) and facilitates the design and semi-synthesis of multicyclic enzymes. These synthetically enhanced durable enzymes add to the current efforts towards a sustainable and green economy.

DOI: $10.1007 / \mathrm{s} 12268-019-1039-5$

(C) Die Autoren 2019

Umweltverschmutzung, Klimawandel, der Verlust der Biodiversität und die Verknappung von Ressourcen sind aktuell dringende Probleme, die jedoch nur langsam von Politik und Wirtschaft als solche angenommen werden. Neue Technologien und Innovationen bieten immer öfter kompromisslose und nachhaltige Alternativen, ohne dabei den bisher gewohn- ten Komfort zu beeinträchtigen. Die green economy hat das Ziel, ökologische Nachhaltigkeit und wirtschaftliche Profitabilität zu verbinden und so den Einsatz erneuerbarer Rohmaterialien und die Verringerung sowie Wiederverwertung von Abfallprodukten zu ermöglichen. Letztendlich wird eine komplett nachhaltige Wirtschaft nur mit neuen Technologien mög-

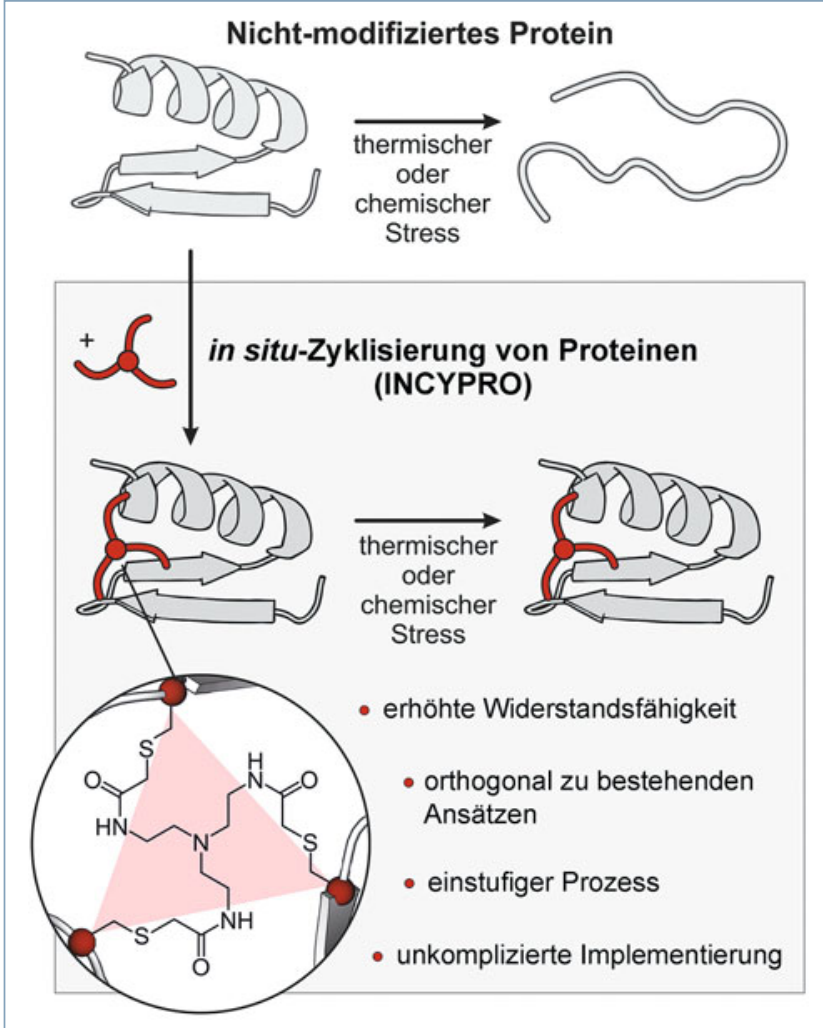

Abb. 1: Konzept der chemisch vermittelten in situ-Zyklisierung von Proteinen (INCYPRO). Drei ausgewählte Aminosäurepositionen an der Proteinoberfläche werden durch Cysteine ersetzt. Die drei eingeführten Thiolgruppen werden dann mit einem triselektrophilen organischen Molekül unter Ausbildung von Thioetherbindungen quervernetzt. Dies führt zu einer Stabilisierung der Tertiärstruktur des Proteins unter thermischem oder chemischem Stress. lich sowie durch den Transfer von einer linearen Wirtschaft hin zu einem zyklischen Aufbau, der vollständig auf die Entstehung von umweltschädlichen Abfallprodukten verzichtet. Die chemische und pharmazeutische Industrie sind Wirtschaftszweige, die entscheidend zu unserem Lebensstandard, aber auch zur Generierung einer Vielzahl von Abfallprodukten beitragen. Außerdem werden in den Herstellungsprozessen häufig Reagenzien eingesetzt, deren Produktion einen hohen Energieverbrauch beinhaltet.

Ein grundlegendes Ziel der chemischen Produktion ist es daher, Reaktionsausbeuten zu erhöhen, umweltfreundlichere Syntheserouten zu etablieren und effizientere katalytische Systeme zu entwickeln - gerade auch mit dem Ziel der Kostenersparnis. Dazu könnten insbesondere die aktuellen Fortschritte in der Biotechnologie beitragen, welche es erlauben, durch den Einsatz von Biokatalysatoren komplexe chemische Reaktionen unter relativ milden Bedingungen durchzuführen [1]. Biokatalysatoren sind beispielsweise einzelne Enzyme oder auch intakte zelluläre Systeme, die es zum Teil schon erlauben, komplexe, mehrstufige chemische Syntheserouten zu ersetzen oder Moleküle $\mathrm{zu}$ erzeugen, die durch chemische Synthese nicht zugänglich sind. Insbesondere der Einsatz von Enzymen oder gekoppelten Enzymsystemen ist biotechnologisch interessant, da diese sich sehr gezielt einsetzen und manipulieren lassen. So gibt es bereits Anwendungen in der Nahrungsmittel-, Textil- und Agrarindustrie sowie bei einer Reihe diagnostischer Verfahren [1]. Für solche Anwendungen ist es häufig notwendig, die Enzyme an deren Wirkungsort anzupassen oder ihr Substratspektrum zu verändern. Wie wichtig derartige Technologien sind, wird durch den diesjährigen Nobelpreis unter anderem für Professor Frances Arnold vom California Institute of Technology, USA, unterstrichen [2]. Sie wurde für ihre Beiträge zur Entwicklung der directed evolution geehrt, durch die es möglich wird, Enzyme durch die Variation der Aminosäuresequenz für eine bestimmte Aufgabe zu optimieren. In vielen Fällen halten jedoch auch diese optimierten Enzyme 


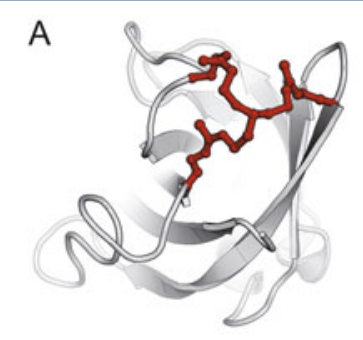

Modell des durch INCYPRO stabilisierten Enyzms Sortase A

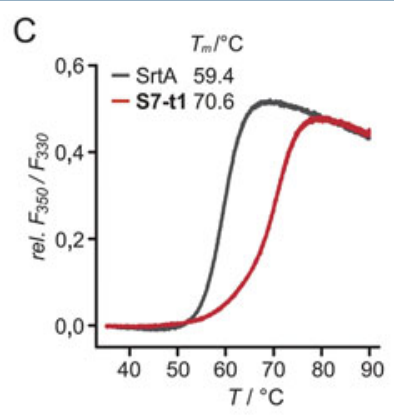

B

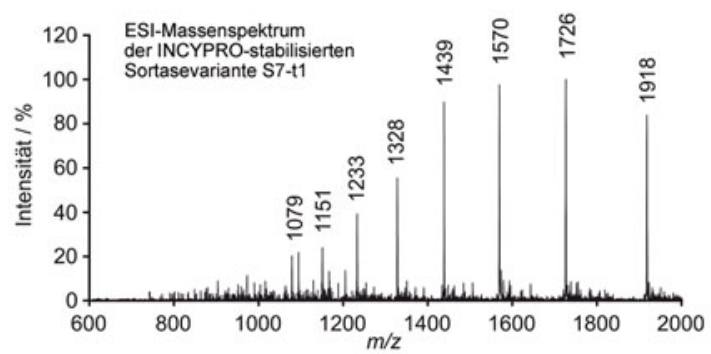

4 Abb. 2: Beispiel der durch INCYPRO stabilisierten Sortase A. A, Modell der molekularen Struktur einer zyklisierten SortaseA-Variante, basierend auf PDB-ID 1t2w. B, ESI-Massenspektrum der durch INCYPRO stabilisierten Sortase S7-t 1 direkt nach der chemischen Quervernetzung. C, Schmelzkurven der Wildtyp-Sortase-A (SrtA, grau) und S7-t1 (rot) einschließlich der resultierenden Schmelzwerte. den harschen Bedingungen, die für eine Produktion in großem Maßstab notwendig sind, nicht stand (z. B. erhöhte Temperaturen, Einsatz von Detergenzien oder nicht-physiologische pH-Werte) [3]. Dies begrenzt den Einsatz von Enzymen in Produktionsprozessen erheblich und stellt eine enorme Hürde [4] für eine vermehrte Verwendung biotechnologischer Verfahren dar.

Wir verfolgen eine Strategie, die es erlaubt, die Robustheit von Enzymen durch die gezielte Modifikation mit kleinen organischen Molekülen zu erhöhen und somit die Vorteile von natürlichen Biokatalysatoren mit den Erkenntnissen der aktuellen Forschung zum Proteindesign und zu biokompatiblen chemischen Reaktionen zu kombinieren [5]. Die unkomplizierte und effiziente Erhöhung der Robustheit von Enzymen ermöglicht einen Einsatz unter extremen Bedingungen (Abb. 1). Diese im letzten Jahr erstmals vorgestellte Technologie beinhaltet die gezielte in situZyklisierung von Proteinen (INCYPRO) durch einen einzelnen nicht-iterativen Designschritt. Dabei werden in einem Computerunterstützten Ansatz, basierend auf den strukturellen Eigenschaften, drei Aminosäurepositionen an der Oberfläche einer Proteindomäne ausgewählt und jeweils durch ein Cystein ersetzt. Unter Umständen ist es nötig, bereits existierende oberflächenexponierte Cysteine im gleichen Schritt aus der Gensequenz zu entfernen. Die resultierende Proteinvariante kann aus den gängigen Expressionssystemen gewonnen werden, da nur proteinogene Aminosäuren zum Einsatz kommen. Im Anschluss werden die Thiolgruppen der drei eingeführten Cysteine mithilfe einer triselektrophilen chemischen Struktur unter der Ausbildung von Thioetherbindungen quervernetzt (Abb. 1, [5]). Die eingeführte Quervernetzung ist dabei chemisch und biologisch inert. Die resultierende multizyklische Proteindomäne weist eine stabile Kernstruktur auf, ohne dass dabei die natürliche Tertiärstruktur verändert wird.

Durch die INCYPRO-Technologie konnten bisher mittels eines einzelnen Modifikationsschritts thermische Stabilisierungen von Proteinen um bis zu $25^{\circ} \mathrm{C}$ erreicht werden [5]. Das Enzym Sortase A bildete dabei ein erstes Modellsystem (Abb. 2A). Für die modifizierte Variante S7-t1 konnte gezeigt werden, dass die chemische Quervernetzung effizient abläuft (Abb. 2B) und mit einer deutlichen Erhöhung der thermischen Stabilität einhergeht (Abb. 2C). Entscheidend für die Anwendbarkeit dieser Technologie ist dabei, dass Enzyme verwendet werden können, die vollständig aus natürlichen Aminosäuren bestehen und somit im Allgemeinen schnell, kostengünstig und in großen Mengen verfügbar sind. Außerdem ist die Enzymoptimierung mittels INCYPRO orthogonal zu bestehenden Stabilisierungsansätzen. Dies ermöglicht ein breites Anwendungsspektrum, da sowohl Wildtyp-Enzyme als auch bereits optimierte Enzyme verwendet werden können. Aufgrund der unkomplizierten Umsetzung und der Langlebigkeit der resultierenden Enzyme kann INCYPRO die derzeitigen Bemühungen hin zu umweltfreundlichen Produktionsverfahren und einer nachhaltigeren Wirtschaft unterstützen. Außerdem ist es denkbar, dass derart robuste Enzyme völlig neuartige biotechnologische Anwendungen in der Abfallbeseitigung oder der Rückführung von Rohstoffen in den Produktionskreislauf ermöglichen.

Derzeit erproben wir die INCYPRO-Technologie an einer Reihe von Enzymen und arbeiten dabei mit der Arbeitsgruppe von Francesco Mutti an der Universität von Amsterdam zusammen, die eine langjährige Expertise bei der Optimierung biotechnologisch relevanter Biokatalysatoren besitzt. Bei unserer Forschung werden wir unter anderem vom Europäischen Forschungsrat durch eine ERC-Proof-of-Concept-Förderung unterstützt. Außerdem wird die Verwertung von INCYPRO über ein universitäres Spin-off vorangetrieben (www.incircular.com).

\section{Danksagung}

Wir danken dem European Research Council und der VU Amsterdam für die Unterstützung. Vielen Dank auch an Dr. Marta Pelay Gimeno für ihre initialen Arbeiten am INCYPRO-Projekt.

\section{Open Access:}

This article is distributed under the terms of the Creative Commons Attribution 4.0 International License (http://creativecommons.org/licenses/by/4.0/), which permits use, duplication, adaption, distribution and reproduction in any medium or format, as long as you give appropriate credit to the original
author(s) and the source, provide a link to the Creative Commons license, and indicate if changes were made. Open access funding provided by Vrije Universiteit Amsterdam, The Netherlands.

\section{Literatur}

[1] Li S, Yang X, Yang S et al. (2012) Technology prospecting on enzymes: application, marketing and engineering. Comput Struct Biotechnol J 2, doi: 10.5936/csbj.201209017

[2] Gibney E, Van Noorden R, Ledford H et al. (2018) 'Testtube' evolution wins Chemistry Nobel Prize. Nature 562:176 [3] Bowler BE (2007) Thermodynamics of protein denatured states. Mol BioSyst 3:88

[4] Bornscheuer UT, Huisman GW, Kazlauskas RJ et al. (2012) Engineering the third wave of biocatalysis. Nature 485:185194

[5] Pelay-Gimeno M, Bange T, Hennig S et al. (2018) In situ cyclization of native proteins: structure-based design of a bicyclic enzyme. Angew Chem Int Ed 57:11164-11170

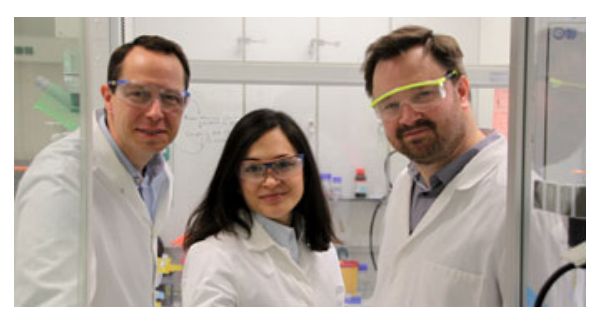

Tom N. Grossmann, Saskia Neubacher und Sven Hennig (v. I. n. r.; Foto: Mathias Wendt)

Korrespondenzadresse:

Prof. Dr. Tom N. Grossmann

Department of Chemistry and Pharmaceutical Sciences

Vrije Universiteit Amsterdam

De Boelelaan 1108

NL-1081 HZ Amsterdam

tom@grossmannlab.com 